\title{
Thermal performance prediction of the TMT optics
}

\section{Myung Cho, Andrew Corredor, Shane Pootrakul, Konstantinos Vogiatzis, George Angeli}

Myung Cho, Andrew Corredor, Shane Pootrakul, Konstantinos Vogiatzis, George Angeli, "Thermal performance prediction of the TMT optics," Proc. SPIE 7017, Modeling, Systems Engineering, and Project Management for Astronomy III, 701716 (16 July 2008); doi: 10.1117/12.789212

SPIE Event: SPIE Astronomical Telescopes + Instrumentation, 2008, Marseille, France 


\title{
Thermal Performance Prediction of the TMT Optics
}

\author{
Myung Cho ${ }^{*}$, Andrew Corredor ${ }^{2}$, Shane Pootrakul $^{2}$, Konstantinos Vogiatzis $^{3}$, George Angeli ${ }^{3}$ \\ ${ }^{1}$ GSMT Program Office, National Optical Astronomy Observatory \\ 950 N. Cherry Ave., Tucson, AZ 85719 \\ ${ }^{2}$ Aerospace and Mechanical Engineering Department, University of Arizona, Tucson, AZ 85721 \\ ${ }^{3}$ TMT Project Office, California Institute of Technology, Pasadena, CA 91125
}

\begin{abstract}
Thermal analysis for the Thirty Meter Telescope (TMT) optics (the primary mirror segment, the secondary mirror, and the tertiary mirror) was performed using finite element analysis in ANSYS and I-DEAS. In the thermal analysis, each of the optical assemblies (mirror, mirror supports, cell) was modeled for various thermal conditions including air convections, conductions, heat flux loadings, and radiations. The thermal time constant of each mirror was estimated and the temperature distributions of the mirror assemblies were calculated under the various thermal loading conditions. The thermo-elastic analysis was made to obtain the thermal deformation based on the resulting temperature distributions. The optical performance of the TMT optics was evaluated from the thermally induced mirror deformations. The goal of this thermal analysis is to establish thermal models by the FEA programs to simulate for an adequate thermal environment. These thermal models can be utilized for estimating the thermal responses of the TMT optics. In order to demonstrate the thermal responses, various sample time-dependent thermal loadings were modeled to synthesize the operational environment. Thermal responses of the optics were discussed and the optical consequences were evaluated.
\end{abstract}

Keywords: thermal models, temperature distributions, thermo-elastic analysis, optical performances

\section{INTRODUCTION}

For large ground-based telescopes, balancing the performance between wind buffeting and mirror and dome seeing has always been one of the challenging requirements to fulfill scientific goals. A two-year environmental measurement of the TMT baseline site ${ }^{[7]}$ and Computational Fluid Dynamics (CFD) techniques provide the thermal environment parameters surrounding the TMT optics. For thermal finite element models, the environmental record and CFD predictions provide expected flow fields and thermal boundary conditions in terms of ambient air profiles, convection coefficients, heat fluxes, and radiation properties. These parameters were implemented to predict thermal responses and thermal deformations of the TMT optics.

The TMT primary segment (M1), a hexagonal mirror, has a thickness of $45 \mathrm{~mm}$, max corner-to-corner length of $1.44 \mathrm{~m}$, and radius of curvature $60 \mathrm{~m}$. The secondary mirror (M2) is a meniscus convex hyperboloid $100 \mathrm{~mm}$ thick, $3.11 \mathrm{~m}$ in diameter, with a radius of curvature $6.42 \mathrm{~m}$. The tertiary mirror (M3) is a flat ellipse with a major axis of $3.512 \mathrm{~m}$, minor axis of $2.508 \mathrm{~m}$, and thickness of $100 \mathrm{~mm}$. The material utilized for the analysis of all the mirrors was a low expansion glass and its physical and material properties are listed in Table 1.

Table 1: Low expansion glass Mirror Properties

\begin{tabular}{|l|c|}
\hline Coefficient of thermal expansion & $30 \times 10^{-9} \mathrm{~m} / \mathrm{m}^{\circ} \mathrm{C}$ \\
\hline Thermal conductivity & $1.46 \mathrm{~W} / \mathrm{m}^{\circ} \mathrm{C}$ \\
\hline Specific heat & $800 \mathrm{~J} / \mathrm{kg}^{\circ} \mathrm{C}$ \\
\hline Density & $2530 \mathrm{~kg} / \mathrm{m}^{3}$ \\
\hline Modulus of elasticity & $9.2 \times 10^{10} \mathrm{~Pa}$ \\
\hline Poisson's ratio & 0.24 \\
\hline Shear modulus & $3.71 \times 10^{10} \mathrm{~Pa}$ \\
\hline
\end{tabular}

*mcho@noao.edu; phone 1520 318-8544; fax 1520 318-8424; www.noao.edu

Modeling, Systems Engineering, and Project Management for Astronomy III, edited by George Z. Angeli, Martin J. Cullum, Proc. of SPIE Vol. 7017, 701716, (2008) · 0277-786X/08/\$18 · doi: 10.1117/12.789212 
In addition, the mirror cell assembly components such as actuators, trusses, fixed frame and rod supports were modeled and utilized for the thermal analysis of the M1 segment in ANSYS. The segment support assembly (SSA) developed by IMTEC is shown in Figure 1(a). Figure 1(b) shows the thermal FE model of the M1 mirror and cell components. The materials used in this thermal analysis were assumed to be titanium for the actuator rod supports and aluminum for all the other components.

An M2 cell assembly (M2CA) design was developed by National Optical Astronomy Observatory (NOAO) as the M2 design and development effort for TMT. The M2CA consists of the M2, M2 supports, M2 control electronic units, and the M2 cell. The M2CA of NOAO is shown in Figure 2(a). Figure 2(b) shows a finite element model of M2CA which is a 60 degree wedge model with a simplified cell and 10 support rods (actuators). This 60 degree FE model was used for initial thermal response calculations, and later it was extended to a full FE model. An M3 cell assembly (M3CA) design was also developed by NOAO. The M3 assembly and a full M3CA FE thermal model are shown in Figures 3(a) and 3(b). The material used for the M2 and M3 support structures was stainless steel. The physical and thermal properties of the mirror support structure materials are listed in Table 2.

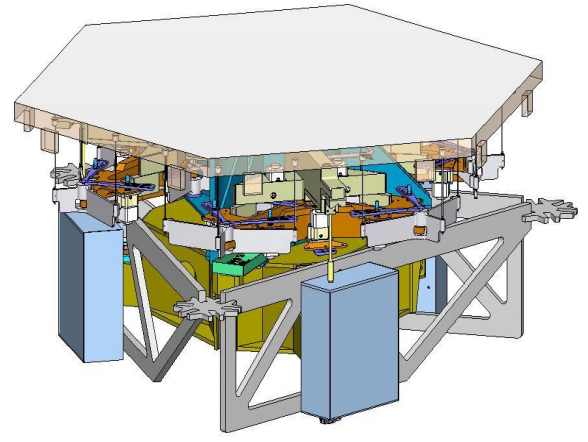

(a)

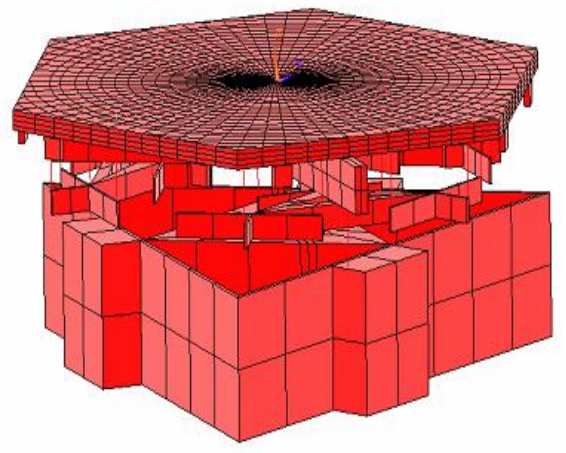

(b)

Fig. 1. M1 and SSA and thermal finite element model. (a) M1 segment and SSA model developed by IMTEC, (b) Segment and SSA thermal FE model

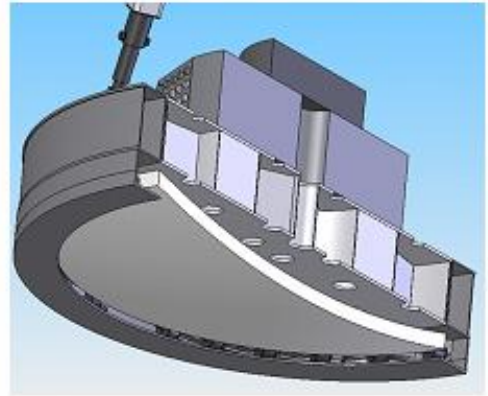

(a)

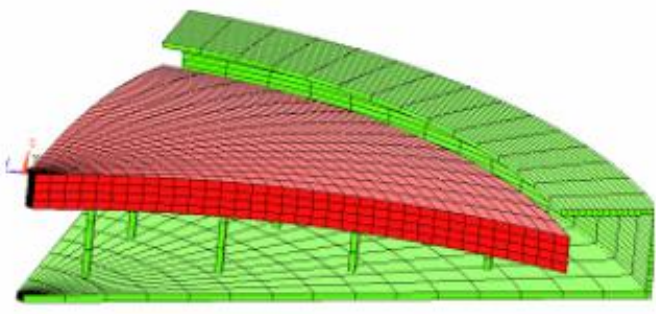

(b)

Fig. 2. Secondary mirror Cell Assembly and an M2CA thermal finite element model. (a) M2 Cell Assembly (M2CA) developed by NOAO, (b) Thermal FE $1 / 6$ model of mirror and support/cell. 


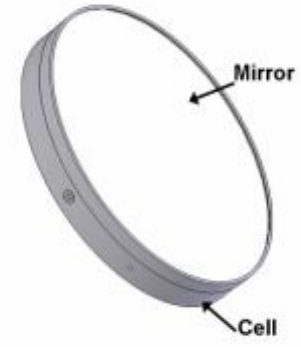

(a)

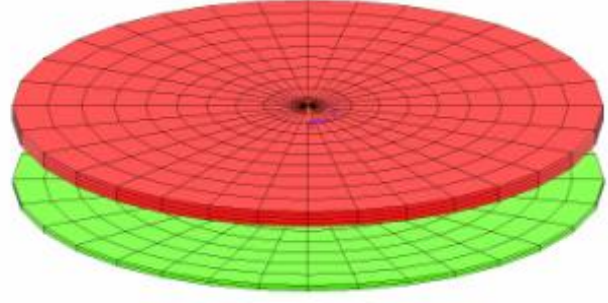

(b)

Fig. 3. Tertiary mirror Cell Assembly and an M3CA thermal finite element model. (a) M3 Cell Assembly (M3CA) developed by NOAO, (b) Thermal FE model of mirror (and cell).

Table 2. M1, M2, and M3 Support Structure Material Properties

\begin{tabular}{|l|c|c|c|}
\hline Material & Aluminum $6061-\mathrm{T} 651$ & Titanium Ti6AI-4V & Stainless Steel T301 \\
\hline Coefficient of Thermal Expansion & $22.8 \times 10^{-6} \mathrm{~m} / \mathrm{m}^{\circ} \mathrm{C}$ & $9 \times 10^{-6} \mathrm{~m} / \mathrm{m}^{\circ} \mathrm{C}$ & $10.8 \times 10^{-6} \mathrm{~m} / \mathrm{m}^{\circ} \mathrm{C}$ \\
\hline Thermal Conductivity & $216 \mathrm{~W} / \mathrm{m}^{\circ} \mathrm{C}$ & $7.6 \mathrm{~W} / \mathrm{m}^{\circ} \mathrm{C}$ & $16.2 \mathrm{~W} / \mathrm{m}^{\circ} \mathrm{C}$ \\
\hline Specific Heat & $917 \mathrm{~J} / \mathrm{kg}^{\circ} \mathrm{C}$ & $2340 \mathrm{~J} / \mathrm{kg}^{\circ} \mathrm{C}$ & $500 \mathrm{~J} / \mathrm{kg}^{\circ} \mathrm{C}$ \\
\hline Density & $2718 \mathrm{~kg} / \mathrm{m}^{3}$ & $4420 \mathrm{~kg} / \mathrm{m}^{3}$ & $8000 \mathrm{~kg} / \mathrm{m}^{3}$ \\
\hline Poisson's Ratio & 0.33 & 0.31 & 0.30 \\
\hline Modulus of Elasticity & $69.65 \times 10^{9} \mathrm{~Pa}$ & $112.0 \times 10^{9} \mathrm{~Pa}$ & $212.0 \times 10^{9} \mathrm{~Pa}$ \\
\hline
\end{tabular}

Thermal environments of the primary segment mirror assembly (M1SA), the M2CA, and the M3CA are similar. The thermal environment of the segment assembly is shown in Figure 4(a). The boundary conditions on the mirror include the absorbed heat flux on the back surface, convection of air on the front surface, convection of air in the back surface, and radiative flux from both front and back surfaces. The basic heat transfer is described with conduction, convection and radiation. Moreover, it is observed that Fourier's equation is satisfied inside the primary segment:

$$
\frac{\partial T}{\partial t}=\alpha \nabla^{2} T
$$

Where $T$ is the mirror's temperature, $t$ is time, and $\alpha$ is the thermal diffusivity given by $\alpha=\frac{k}{\rho C_{p}}$, where $k$ is the thermal conductivity, $\rho$ is the density, and $C_{p}$ is the specific heat of the mirror.

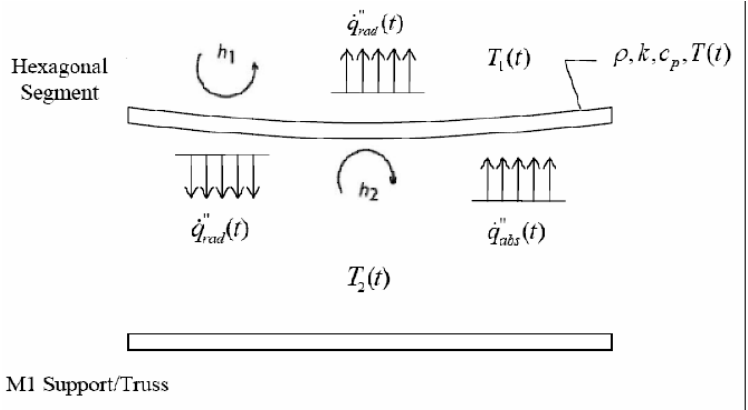

(a)

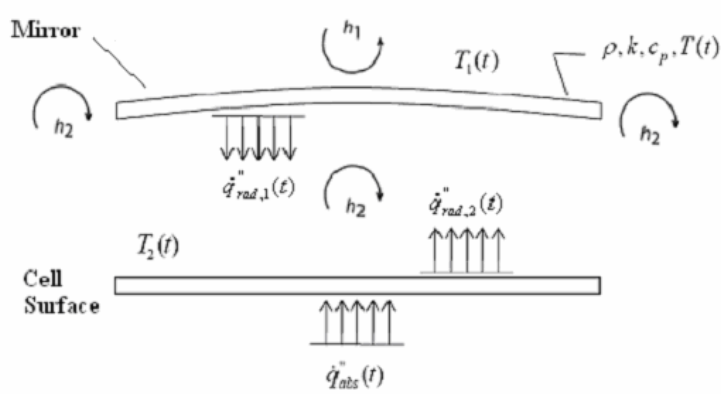

(b)

Fig. 4. (a) Thermal environment of M1CA; (b) Thermal environment of M2CA, and is also applicable to M3CA. 
The boundary condition on the front surface is given by

$$
\dot{q}_{\text {rad }}^{\prime \prime}(t)+\dot{q}_{\text {conv }}^{\prime \prime}(t)=\dot{q}_{\text {cond }}^{\prime \prime}(t) ; \text { or, } \quad \dot{q}_{\text {rad }}^{\prime \prime}(t)+h_{1}\left[T(\text { front })-T_{1}(t)\right]=-\left.k \frac{\partial T}{\partial n}\right|_{\text {front }}
$$

Similarly, the boundary condition on the back surface is

$$
\dot{q}_{a b s}^{\prime \prime}(t)-\dot{q}_{r a d}^{\prime \prime}(t)-\dot{q}_{c o n v}^{\prime \prime}(t)=-\dot{q}_{c o n d}^{\prime \prime}(t) ; \text { or, } \dot{q}_{a b s}^{\prime \prime}(t)-\dot{q}_{r a d}^{\prime \prime}(t)-h_{2}\left[T(\text { back })-T_{2}(t)\right]=\left.k \frac{\partial T}{\partial n}\right|_{b a c k}
$$

Where

$\dot{q}_{\text {rad }}^{\prime \prime}(t)$ : Time-dependent radiative flux from the front surface

$\dot{q}_{\text {conv }}^{\prime \prime}(t)$ : Air convection in the front surface

$h_{1} ; h_{2}:$ Heat transfer coefficient in the front surface and the back surface, respectively

$T_{1}(t) ; T_{2}(t)$ : Air temperature in front and back of the mirror, respectively

$n$ : vector normal to the mirror surface

These boundary conditions vary slightly among the mirror assemblies depending on the heat loads and thermal sources. Similarly, the boundary conditions for M2CA and M3CA are shown in Figure 4(b). The boundary condition equations satisfying M2CA and M3CA are determined in the same manner as M1SA with three exceptions: (1) only radiation between the mirror and cell is considered and not to the atmosphere, (2) convection is added to the mirror edges, and (3) heat flux is applied to the cell back instead of the mirror back.

By using finite element methods, detailed analyses have been performed on each of the TMT mirror assemblies. The thermal responses from the FE models were calculated and the time history results were recorded in a 24 hour heat cycle. Thermal performance evaluations of the mirrors and their assemblies have been investigated under various thermal environments. These simulation models allow assessment of the merits of various thermal loads and boundary conditions surrounding the mirror assemblies to be assessed. For each of these thermal environments, the thermal input, the responses, and thermo-elastic results were reported in references [2], [4], and [5]. Hence, this paper focuses on the thermal loads, boundary conditions, thermal responses, and the optical surface errors only for the latest thermal environment scenario. The detailed descriptions are addressed in the following chapters.

\section{THERMAL ANALYSIS OF M1 SEGMENT ASSEMBLY (M1SA)}

\subsection{Thermal Loads and Boundary Conditions}

In order to evaluate the thermal response of the segment assembly for a realistic thermal environment, reasonably well defined telescope operation boundary conditions were assumed for the entire segment assembly. The conditions were air convection on the front and back surfaces of the segment as well as air convection on the surface of the support hardware component, and heat generation from the actuators and pre-amps. Furthermore, various analyses were conducted in order to clarify the thermal results. The analyses performed were as follows:

- Case A: Air convection was the only applied thermal load.

- Case B: Heat generation was the applied thermal load (Radiation).

- Case C: Combined thermal loads (Case A and Case B).

\subsubsection{Air temperature variation}

Extensive CFD analyses have been performed to quantify air flows inside the enclosure. Moreover, some simulation schemes have been developed for the air temperature distribution around the optics. For the current analysis, a timedependent air temperature was assumed to have a linear variation. During a 24 hour cycle, the maximum air temperature difference relative to the temperature of the mirror was assumed to be $2{ }^{\circ} \mathrm{C}$ as shown in Figure 5 (a). In the analysis over 
the 24 hour thermal cycle period, the applied temperature loads were assumed to be absolute, rather than a temperature difference between the mirror and ambient conditions. It was further assumed that a time of zero hours indicates sunset or the beginning of night. The air temperature was applied on both front and back surfaces of the segment, the surface of mirror cell components, edge sensors and pre-amps.

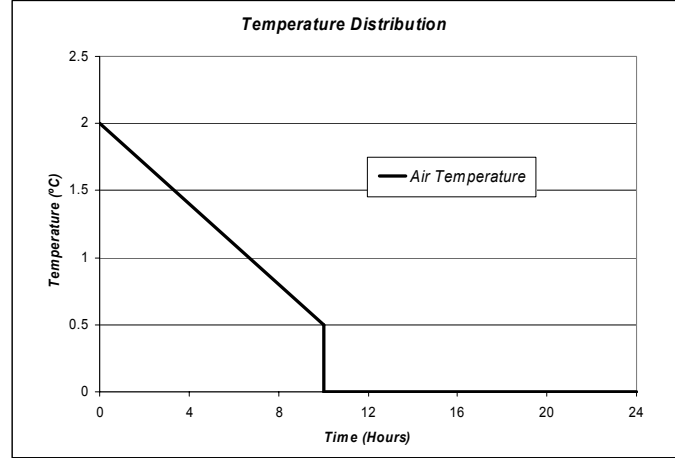

(a)

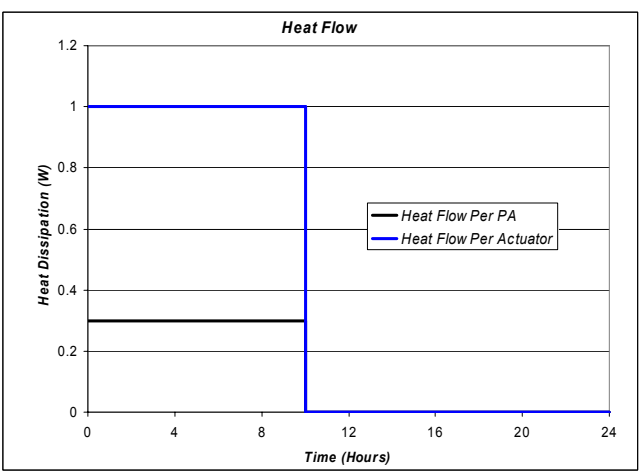

(b)

Fig. 5. Thermal loads, (a) Ambient air temperature profile, (b) Heat flow profiles of pre-amps and cell actuators.

\subsubsection{Heat Generation (Radiation)}

The segment will absorb heat on the back surface due to radiation coming from the three heat generating actuators and the six heat generating pre-amps. The heat dissipated by each of the actuators is $1 \mathrm{~W}$ and the heat dissipated by each preamp is $0.3 \mathrm{~W}$ as shown in Figure 5(b). It was further assumed that the heat flow was constant for the first 10 hours with a maximum of $1 \mathrm{~W}$ for the actuators and $0.3 \mathrm{~W}$ for the pre-amps. The heat sources are equivalent to uniform heat generation rates of $110.2 \mathrm{~W} / \mathrm{m}^{3}$ per actuator and $1128.5 \mathrm{~W} / \mathrm{m}^{3}$ per pre-amp. These six pre-amps were located at 50 percent the distance along the radius and separated by 60 degrees. Currently, the analysis assumes that they are mounted on the back of the mirror segment. However, the pre-amps will be relocated to the segment support assembly (SSA) to reduce the thermal interactions with the mirror segment.

\subsection{Finite Element Models}

In order to calculate the temperature distribution of the TMT M1 assembly, a finite element of the entire mirror assembly was generated using ANSYS. The hexagonal segment was modeled with 4 layers of thermal elements (SOLID70) and shell elements (SHELL57) were created on its front and back surfaces to facilitate the application of thermal loads. In addition, the mirror cell components, the edge sensors, and pre-amps were modeled using thermal elements (SOLID 70). Shell elements (SHELL57) were created on their surfaces, as well, in order to apply thermal loads. Some components in the Segment Support Assembly (SSA) which are not thermally significant were excluded from the FE thermal models for computational efficiency. Finally, a super-element (MATRIX50) was used in order to perform the radiation analysis between surfaces (segment and components). The finite element model used in this analysis is shown in Figure 1(b). By conducting a thermal analysis in ANSYS, the thermal time constant and the temperature distribution for the different cases described in section 2.1 were calculated.

\subsection{Thermal Analysis Results}

For the thermal time constant, a convection analysis was performed with a segment thermal FE model. An air convection was applied on the front and back surfaces having heat transfer coefficients of $5 \mathrm{~W} / \mathrm{m}^{2}{ }^{\circ} \mathrm{C}$ and $1 \mathrm{~W} / \mathrm{m}^{2}{ }^{\circ} \mathrm{C}$, respectively. Air convection along the edge of the segment was disregarded. The convection coefficient of $5 \mathrm{~W} / \mathrm{m}^{2}{ }^{\circ} \mathrm{C}$ on the front surface is approximately equivalent to a wind velocity of $1 \mathrm{~m} / \mathrm{sec}$ inside the enclosure based on a CFD analysis. The thermal time constant $(\tau)$ of 5 hours was calculated by obtaining the required time for the mirror to reach $3.7^{\circ} \mathrm{C}$ as $1 / \mathrm{e}$ of the initial temperature at $10^{\circ} \mathrm{C}$. The thermal responses of the convection case (Case A) show the peak temperature reached $0.97^{\circ} \mathrm{C}$ at 6 hours. For the heat generation case (Case B), a radiation analysis was performed using the uniform 
heat flow shown in Figure 5(b). Due to radiation, the front surface reached the maximum temperature of $0.45{ }^{\circ} \mathrm{C}$. Detailed thermal responses for both Case A and Case B were discussed in references [2] and [4].

This paper addresses the most recent test scenario, Case C: Combined thermal loads, using the extended model. The temperature distribution of the mirror was calculated by combining the thermal loads used in the previous cases (air convection and radiative heat generation). The thermal response was plotted over time using nodes from the front surface and back surface of the mirror positioned at a pre-amp location. The peak temperature responses obtained were $2.21{ }^{\circ} \mathrm{C}$ and $1.30{ }^{\circ} \mathrm{C}$ on the back and front surface, respectively, occurring at 7.5 hours. Therefore, the temperature gradient at the peak response was $0.914{ }^{\circ} \mathrm{C}$ through the thickness of the mirror as shown in Figure 6(a). The temperature distribution at the peak of the mirror back surface is shown in Figure 6(b). This figure vividly shows the foot-print of six pre-amps in red and three actuators in green. Additionally, the effects of conduction through the rod flexures were investigated. From the radiation analysis it was observed that conduction through the rod flexures was insignificant since their conductive areas are small compared to other components.

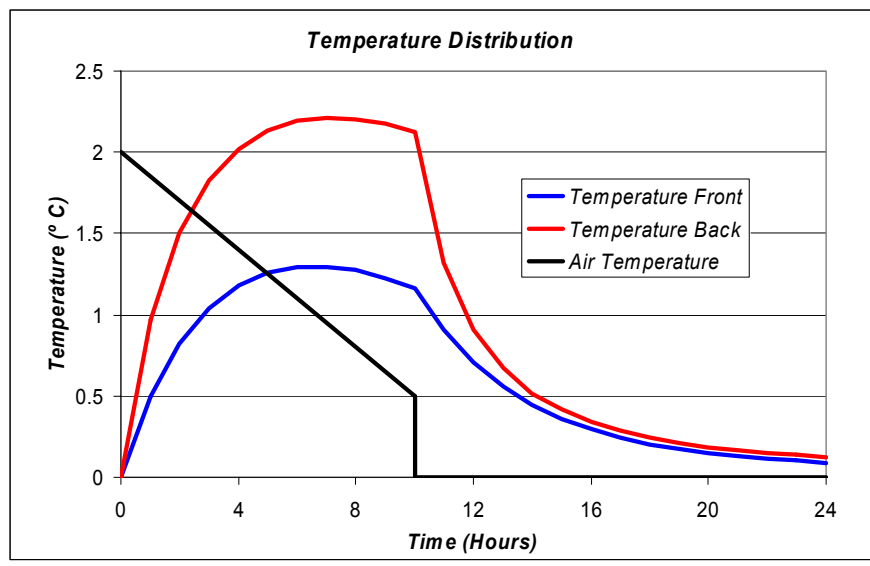

(a)

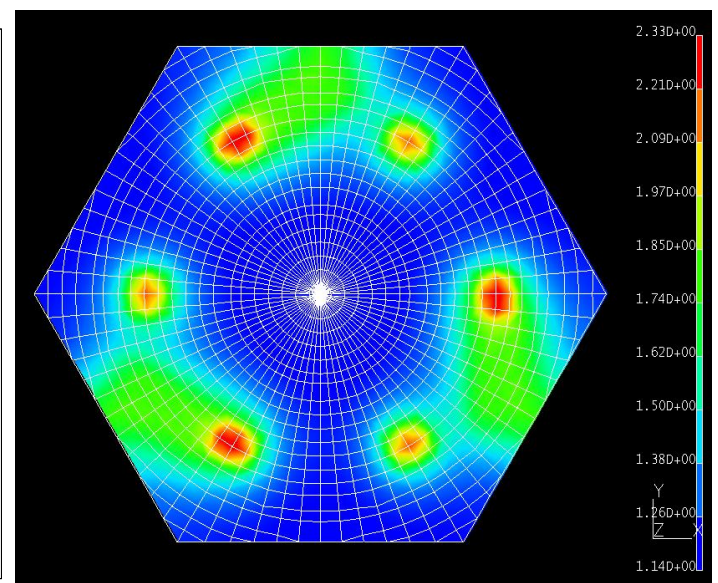

(b)

Fig. 6. Temperature distributions: (a) Temperature distribution from combined case, (b) Temperature distribution on the mirror back surface at 7.5 hours.

\subsection{Thermo-elastic Analysis}

The thermal deformation of the hexagonal segment was calculated using the thermal response from the peak temperature during the 24 hour thermal cycle. From the thermal analysis performed in Case $\mathrm{C}$ (combined loads), the nodal temperature at 7.5 hours was applied to the segment model in order to perform the thermo-elastic analysis. Furthermore, the thermal elements in ANSYS, SOLID70 and SHELL57, were converted to structural elements SOLID45 and SHELL41, respectively.

For the thermo-elastic analysis, the model was constrained by three points on the back surface. These supports were located at 67 percent the distance along the radius and separated by 120 degrees. A kinematic 3-point constraint was used for the boundary conditions. From the thermo-elastic analysis the segment deformation was obtained. A peak-valley surface error of $4.6 \mathrm{~nm}$ and RMS surface error of $0.3 \mathrm{~nm}$ was calculated over the optical surface. This optical surface can be further improved by correcting piston, tilts, and focus (PTF) aberrations. This correction can be achieved either by M1 active optics or other compensation Look-Up Table. The optical surface maps of the maximum thermal response before and after corrections are shown in Figure 7. The optical surface RMS error of $0.3 \mathrm{~nm}$ was reduced to $0.1 \mathrm{~nm}$ after corrections. 


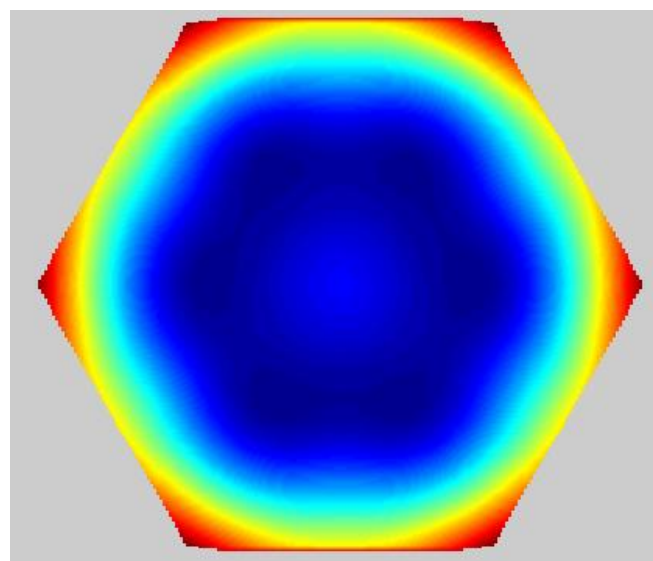

(a)

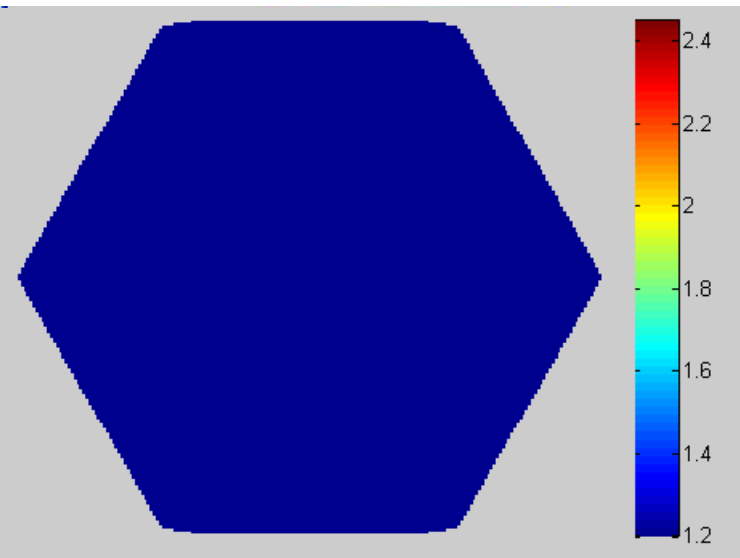

(b)

Fig. 7. (a) Thermal deformation calculated over the entire optical surface before PTF corrections, (b) the optical thermal deformation after PTF corrections (units in $\mathrm{nm}$ ).

\section{THERMAL ANALYSIS OF M2 ASSEMBLY (M2CA)}

\subsection{Thermal Loads and Boundary Conditions}

In order to evaluate the thermal response of the secondary mirror, various thermal loads and boundary conditions were considered. Three testing environment cases were performed on the secondary mirror by itself. Seven thermal environment cases, four unit loads and three combined loads, were performed using a simplified full thermal FE model of the M2 cell assembly (M2CA).

The first three test environments (mirror only) were used to determine the thermal time constant of the mirror, the effects of convection on the mirror, and the effects of radiation cooling from the mirror to a "cold plate". The remaining seven thermal conditions applied to the extended model were used to investigate the effects of other components on the secondary mirror. This extended model will represent a more realistic thermal environment. The thermal conditions on the extended model are listed in Table 3. This paper addresses the most recent test scenario, Case 7; the other thermal loads and boundary condition cases are described in more detail in reference [5]. All test environments were performed over a thermal cycle period of 24 hours, where a time of zero hours indicates sunset or the beginning of night. Additionally, the applied temperature loads were assumed to be absolute, not a temperature difference between the mirror and ambient conditions.

Table 3. Extended M2CA thermal model boundary conditions

\begin{tabular}{|c|l|}
\hline Case & \multicolumn{1}{c|}{ Boundary Condition } \\
\hline $\mathbf{1}$ & Convective cooling on mirror and cell surfaces with conduction through rods \\
\hline $\mathbf{2}$ & Convective heating on mirror and cell surfaces with conduction through rods \\
\hline $\mathbf{3}$ & Sinusoidal Heat flux into cell back with conduction through rods \\
\hline $\mathbf{4}$ & Surface-to-surface radiation due to sinusoidal heat flux into cell back surface \\
\hline $\mathbf{5}$ & Combined boundary conditions with convective cooling, no cold plate \\
\hline $\mathbf{6}$ & Combined boundary conditions with convective heating, sinusoidal heat flux \\
\hline $\mathbf{7}$ & Combined boundary conditions with convective heating, constant heat flux \\
\hline
\end{tabular}

\subsubsection{Air temperature variation (Convection)}

The air temperature used for the convective thermal load was assumed to be warmer than the temperature of the mirror cell. The convection was assumed to have a linear variation in air temperature as shown in Figure 5(a). Based on Figure 4(b), this analysis assumes that the convection coefficient $h_{1}$ on the exposed mirror surface was $3 \mathrm{~W} / \mathrm{m}^{2}{ }^{\circ} \mathrm{C}$ and $h_{2}$ on the 
enclosed surfaces was $1 \mathrm{~W} / \mathrm{m}^{2}{ }^{\circ} \mathrm{C}$. The heat transfer coefficient of $3 \mathrm{~W} / \mathrm{m}^{2}{ }^{\circ} \mathrm{C}$ on the front surface is approximately equivalent to a wind velocity of $1 \mathrm{~m} / \mathrm{sec}$ inside the enclosure based on a CFD analysis.

\subsubsection{Heat Generation (Radiation)}

The secondary mirror cell will absorb heat on the cell's back surface due to heat generation from the actuators and electronic components. This is the only assumed heat source for the secondary mirror, providing direct heat flux into the support structure's cell wall back and radiation exchange between the mirror and cell wall surfaces. The heat generated was assumed to provide a constant $2 \mathrm{~W} / \mathrm{m}^{2}$ of incident heat flux on the cell's back surface for the first ten hours of the twenty-four hour thermal cycle. This heat flux represents the assumed operation and idle time of the telescope, as shown in Figure 8. This constant heat flux can be further extended to simulate a full 24 hour operation scenario if applicable.

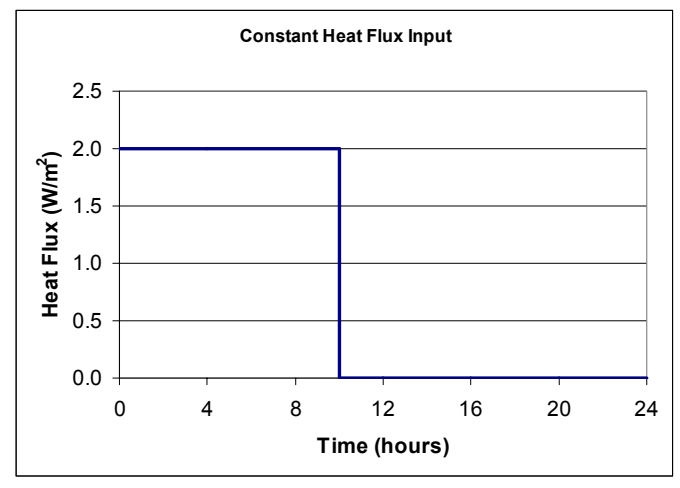

Fig. 8. Heat flux from electronics into M2 and M3 cell back wall.

\subsection{Finite Element Models}

There were three different finite element models utilized for the analysis in ANSYS. The first model was a simple 60 degree wedge of the mirror itself. The second model was an extended version of the first with a simplified cell and support structure, as shown in Figure 2(b). The final variation was a simplified, but complete 360 degree model, similar to the one shown in Figure 3(b) for the tertiary mirror. It was necessary to simplify the final variation more than the second model due to computational limitations and efficiency due to the increase in element numbers. Based on investigation and the results of the first two models, the following four assessments were made to achieve the final model. First, conduction through the support rods is negligible due to a small cross-sectional area. Additionally, the invar material joining the mirror to the support rods further limits conductive effects. Second, conduction up the sides of the cell wall is negligible due to a high conductive resistance in the direction of heat flow. Third, due to limitations in ANSYS, symmetry conditions are not supported for surface-to-surface radiation; therefore, a 60 degree wedge is not accurate. Lastly, radiation exchange between the cell wall sides and mirror sides is negligible due to the second assumption and the relatively small surface areas of interaction. In other words, convection dominates, and only the area of the mirror's back surface is large enough for radiation to affect mirror performance during operation.

The ANSYS FE models were created using the thermal elements SOLID70, LINK33, SHELL57, and MATRIX50. SOLID70 was used to create the four element mirror layers and the single layer cell frame. LINK33 was used to simulate conduction through the support rods from the cell plate to the mirror's back surface. SHELL57 was used to calculate the view factors between the radiating surfaces. Finally, MATRIX50 was used to implement the calculated view factors and simulate the surface-to-surface radiation effects.

\subsection{Thermal Analysis Results}

For the thermal time constant, a convection analysis was performed with the M2 thermal FE mirror-only model. An air convection was applied on the front and back surfaces having heat transfer coefficients of $3 \mathrm{~W} / \mathrm{m}^{2}{ }^{\circ} \mathrm{C}$ and $1 \mathrm{~W} / \mathrm{m}^{2}{ }^{\circ} \mathrm{C}$, 
respectively. Air convection along the edge of the mirror was incorporated. The thermal time constant $(\tau)$ of 14 hours was calculated by obtaining the required time for the mirror to reach $3.7^{\circ} \mathrm{C}$ as $1 / \mathrm{e}$ of the initial temperature at $10^{\circ} \mathrm{C}$.

This paper addresses the most recent test scenario, Case 7 listed in Table 3, using the extended model. Results and responses of the other thermal loads and boundary condition cases are described in more detail in reference [5]. The thermal response of the latest thermal loads and boundary conditions was performed. The results obtained for the TMT secondary mirror with constant heat flux, surface-to-surface radiation exchange, and warm convection, are shown in Figure 9(a). The temperature time history was plotted at four points along the radius on the front and back mirror surfaces, starting at $0 \%$ (mirror center) to $100 \%$ (mirror outer edge). For example, "front $50 \%$ " represents the radial zone halfway between the center and edge on the exposed front mirror surface and "back $100 \%$ " represents the temperature along the mirror's edge on the enclosed back surface. The results show the maximum temperature reached for this test case was approximately $0.65^{\circ} \mathrm{C}$. It can be seen from Figure 9(a) that the general shapes of the temperature distributions for the secondary mirror are similar to those for the M1 segment in Figure 6(a), despite the differences in mirror shape and applied heat flux sources. These results indicate that the dominant heat transfer mode is convection. This is expected since the temperature difference between the radiating surfaces is too small for radiation to be dominant in a combined mode heat transfer scenario. Neglecting the convection edge effects, the temperature distribution across the mirror surface is relatively uniform.

It is of interest to determine the temperature differences (delta) between the secondary mirror's front and back surfaces over the 24 hour cycle for the combined case with warm air, constant heat flux, and surface-to-surface radiation. In this study, a goal temperature difference range of $0.10^{\circ} \mathrm{C}$ was targeted. The temperature delta for the discussed case is shown in Figure 9(b). There are two peaks that occur, one at 1.5 hours and a second at 11.0 hours. The peak occurring at 1.5 hours indicates the exposed front surface temperature is warmer than the back enclosed surface by approximately $0.095^{\circ} \mathrm{C}$. Conversely, the dip at 11.0 hours indicates the back surface is warmer than the front surface by $0.065^{\circ} \mathrm{C}$. It is apparent that the $h_{1}$ and $h_{2}$ convection coefficients applied on the M2 surfaces dictates the general shape of the temperature delta. The shape resembles the applied load shown in Figure 5(a), further supporting that convection is the dominant heat transfer mode. Neglecting the edge effects, the temperature delta across the surface is relatively uniform.

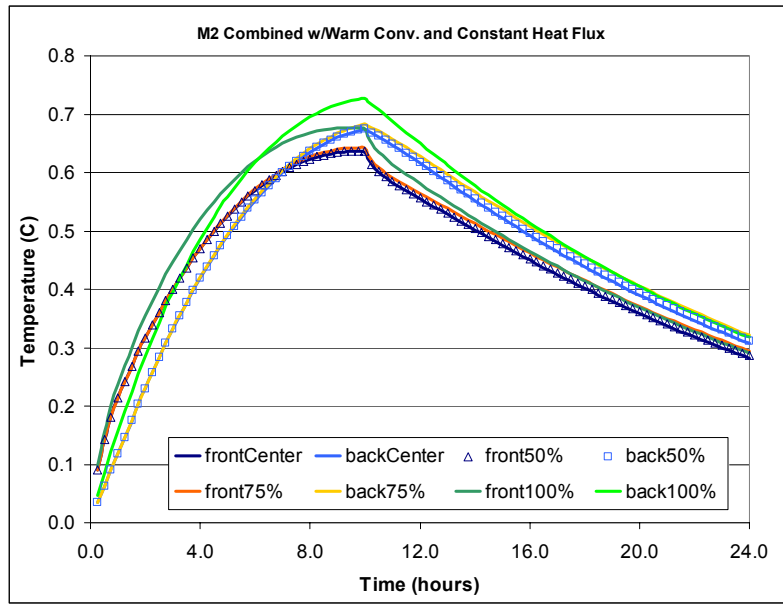

(a)

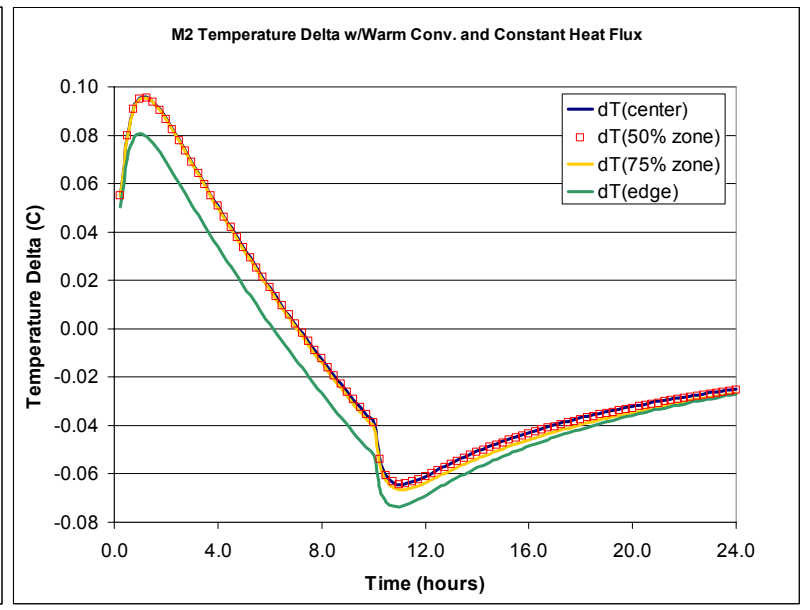

(b)

Fig. 9. (a) Temperature distributions at four radial locations over the M2 for Case 7 (warm air, constant heat flux, and surface-to-surface radiation); (b) Delta temperature at the four radial locations.

\subsection{Thermo-elastic Analysis}

The thermal response analysis performed on the TMT secondary mirror was used to obtain a thermo-elastic deformation. The thermo-elastic deformations of interest occur at the point in time of maximum front-to-back surface temperature difference. This front-to-back surface temperature difference commonly contributes to the overall focus change of the mirror. Therefore, displacements along the optical axis (the z-direction in the FE models) are of interest for all analyses. 
This paper addresses the most recent test scenario, Case 7 listed in Table 3. The temperature distribution from the latest thermal loads and boundary condition was applied to evaluate the optical thermal deformation. A kinematic 3-point constrained was applied on the secondary mirror around the $20 \%$ radial zone from the center. The maximum zdisplacement is $7.2 \mathrm{~nm}$ from the thermal deformation as shown in Figure 10(a). The deflection is in the negative direction, toward the cell structure. This is in agreement with the temperature delta results; the warmer front mirror surface is more expanded than its relatively cooler back mirror surface. The maximum displacement vector magnitude ( $\mathrm{x}-, \mathrm{y}-$, and $\mathrm{z}$-direction), was found to be $11.3 \mathrm{~nm}$ toward the cell structure. The maximum thermo-elastic response can be further improved by correcting piston, tilts, and focus aberrations. This correction can be achieved either by M2 active optics or other compensation Look-Up table. The optical surface maps of the maximum response before and after the corrections are shown in Figure 10(b) and 10(c) when M2 is at a face up position. The optical surface RMS errors were calculated over the entire optical surface. Before corrections, the maximum peak-to-valley (PV) distance was $7.2 \mathrm{~nm}$ with an RMS error of $2.2 \mathrm{~nm}$. After corrections, the maximum PV distance was $1.2 \mathrm{~nm}$ with an RMS error of only $0.3 \mathrm{~nm}$. The thermal response calculations assume $\mathrm{M} 2$ is at a face up position.

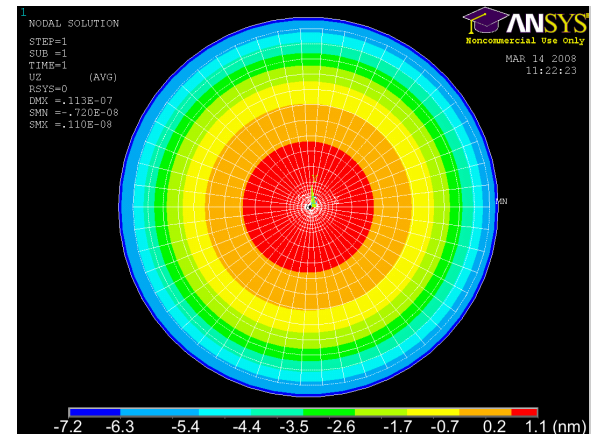

(a)

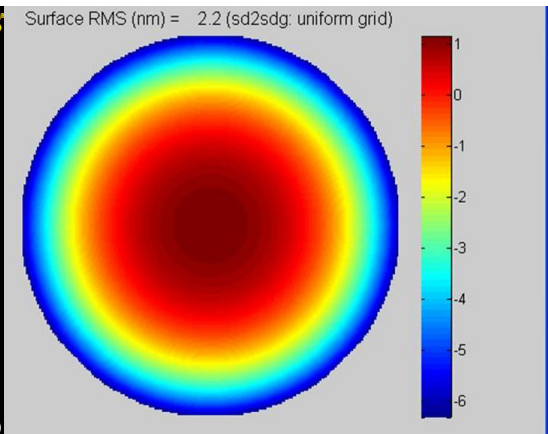

(b)

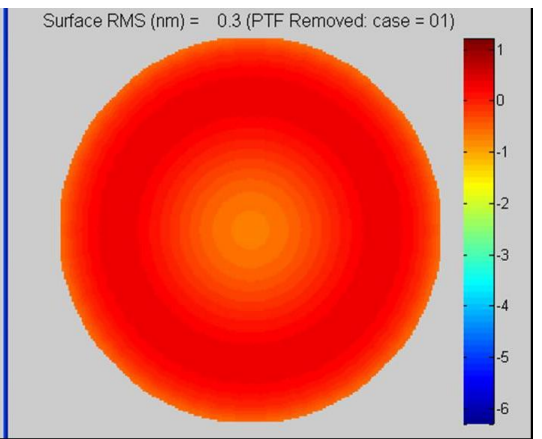

(c)

Fig. 10. (a) thermo-elastic deformation - combined, warm, constant heat flux at 1.5 hours, (b) thermal deformation calculated over the entire optical surface before PTF corrections, (c) the optical thermal deformation after PTF corrections.

\section{THERMAL ANALYSIS OF M3 ASSEMBLY (M3CA)}

\subsection{Thermal Loads and Boundary Conditions}

All thermal loads and boundary conditions applied to the tertiary mirror are the same as those applied to the secondary mirror with a few exceptions. Based on the similarities between the secondary and tertiary mirrors, the analysis was only performed for an extended, simplified tertiary cell model. Since it was found that conduction through the support rods was negligible for the secondary mirror, test cases 1, 2, and 3 from Table 3 were omitted for the tertiary mirror. Additionally, test case 5, combined with cooling convection, was modified to include radiation cooling to a cold plate behind the tertiary mirror. Details for these boundary conditions and scenarios are described in [5]. It is also important to note that the convection coefficients applied on M3CA follow the same rules as those for M2CA. All participating surfaces enclosed within the cell structure were assigned a convection coefficient of $h=1 \mathrm{~W} / \mathrm{m}^{2 \circ} \mathrm{C}$ while the exposed front surface was assigned a convection coefficient of $h=3 \mathrm{~W} / \mathrm{m}^{2}{ }^{\circ} \mathrm{C}$, which corresponds to a wind speed of $1 \mathrm{~m} / \mathrm{s}$.

\subsection{Finite Element Models}

As mentioned in the previous section, all analyses for the tertiary mirror were performed for the extended cell model. The simplified final elemental tertiary mirror cell model consisted of the mirror and back plate, similar to the secondary mirror. The model was created based on the results and findings of the secondary mirror analysis, recalling that conduction through the support rods and cell side walls was negligible. The finite element model is shown in Figure $3(b)$; the cell is represented by the green elements. 


\subsection{Thermal Analysis Results}

For the thermal time constant, a convection analysis was performed with a M3 thermal FE model. An air convection was applied on the front and back surfaces having heat transfer coefficients of $3 \mathrm{~W} / \mathrm{m}^{2}{ }^{\circ} \mathrm{C}$ and $1 \mathrm{~W} / \mathrm{m}^{2}{ }^{\circ} \mathrm{C}$, respectively. Air convection along the edge of the M3 was also included. The thermal time constant $(\tau)$ of 14 hours was calculated by obtaining the required time for the mirror to reach $3.7^{\circ} \mathrm{C}$ as $1 / \mathrm{e}$ of the initial temperature at $10^{\circ} \mathrm{C}$.

The latest thermal loads and boundary condition for the tertiary mirror was a combined case of warm convection with constant heat flux and surface-to-surface radiation. The thermal response shows similar results to those in Figure 9 for M2CA. Neglecting the edges, which show the highest temperatures due to an added convection effect on the sides, the maximum temperature reached for this test case was approximately $0.57^{\circ} \mathrm{C}$, as seen in Figure 11 (a). Additionally, the shapes of the curves are the same as in the previous section despite the differences in mirror shape between the M2 and the M3. These results further support that convection, although small, is still the dominant mode of heat transfer.

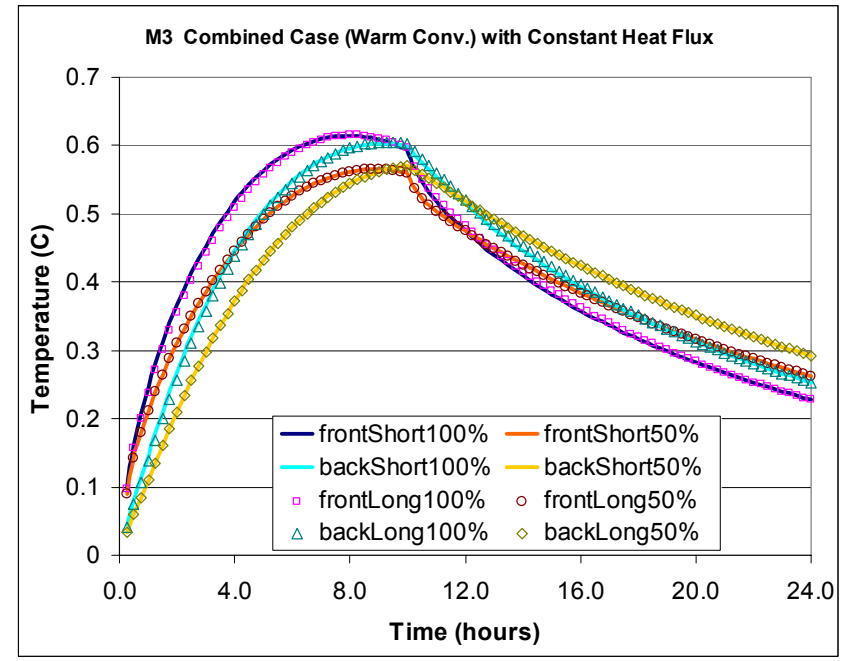

(a)

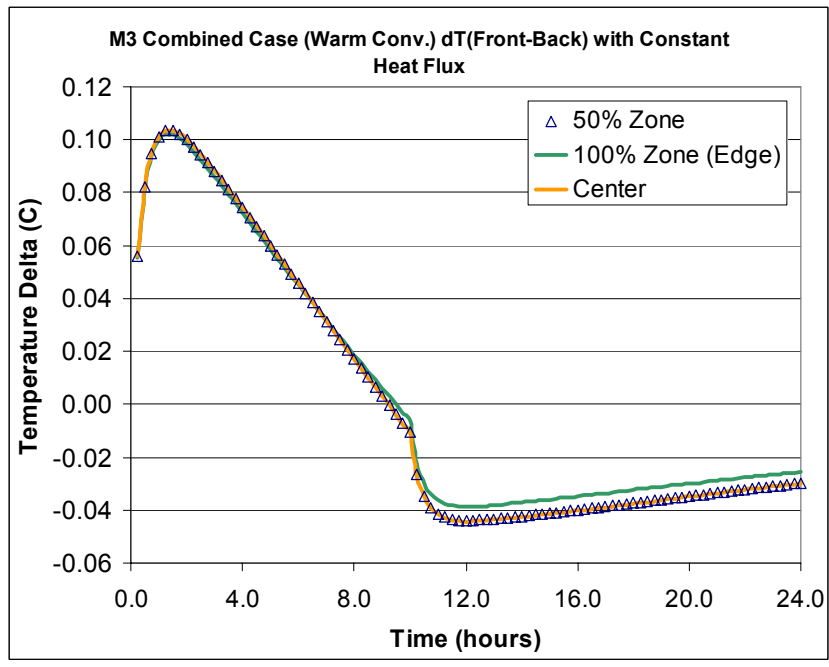

(b)

Fig. 11. (a) Temperature distributions at four radial locations over the M3 for combined case (warm air, constant heat flux, and surface-to-surface radiation); (b) Delta temperature at two radial locations on the major axis.

The temperature delta between the front and back surfaces for the tertiary mirror with warm convection, constant heat flux, and surface-to-surface radiation are shown in Figure 11(b). The results are similar to those for the secondary mirror, following the form of the applied warm convection thermal load. The maximum temperature differences are $0.10^{\circ} \mathrm{C}$ at 1.5 hours and $0.045^{\circ} \mathrm{C}$ at 11.5 hours.

\subsection{Thermo-elastic Analysis for Latest Combined Loads}

This paper addresses the most recent test scenario using the extended model. Results and responses of the other thermal loads and boundary condition cases are described in more detail in reference [5]. The temperature distribution from the latest thermal scenario was applied to evaluate the M3 thermal deformation. For the thermo-elastic deformation calculations, the temperature distribution at the point in time of maximum front-to-back surface temperature difference at 1.5 hours was utilized. As with M1SA and M2CA, M3CA also utilized a 3-point kinematic constraint.

The results from the M3 thermo-elastic analysis are shown in Figure 12. This tertiary mirror model was enhanced for effective post processing by providing a more uniform surface node distribution. The maximum displacement magnitude was $48.5 \mathrm{~nm}$. The maximum z-displacement, normal to the mirror surfaces, was $-47.4 \mathrm{~nm}$ at a time of 1.5 hours, as seen in Figure 12. 


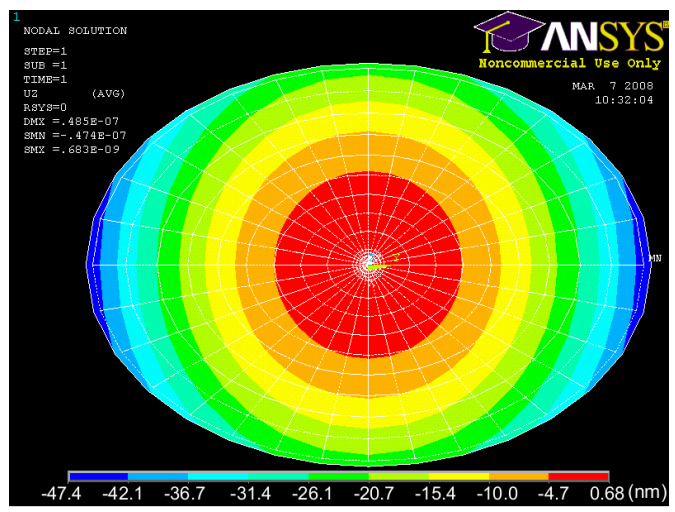

Fig. 12. M3 deformation at 1.5 hours for the combined case (warm convection, surface radiation, constant heat flux).

The optical surface deformation was calculated. The resulting surface is shown in Figure 13(a) when M3 is at a face up position. The optical surface RMS error of $11.3 \mathrm{~nm}$ was calculated over the entire optical surface. For the 15-minute field of view (FOV), nine sub-apertures were constructed to simulate the thermal print-through effects over the M3 for the multiple instrument locations. The surface map of each of the nine sub-apertures for the print-through and the RMS surface errors are shown in Figure 13(b). This optical surface error can be further improved by correcting piston, tilts, and focus (PTF) aberrations. Corrections can be achieved either by M3 active optics or other compensation Look-Up table. The optical surface map after corrections is shown in shown in Figure 13(c). This demonstrates that the thermal deformation can be corrected almost entirely by the M3 active optics.

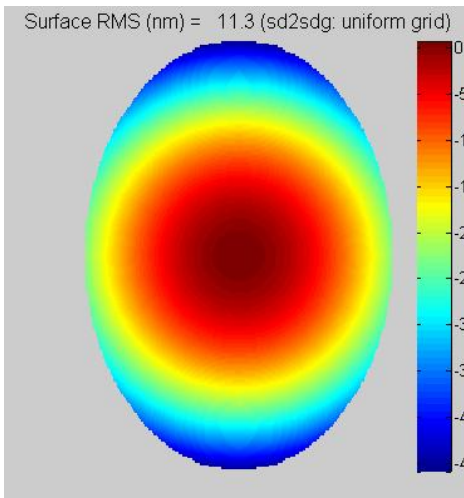

(a)
$\mathrm{RMS}=10.2 \mathrm{~nm}$

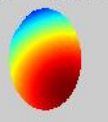

$\mathrm{RMS}=6.9 \mathrm{~nm}$

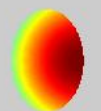

$\mathrm{RMS}=10.1 \mathrm{~nm}$

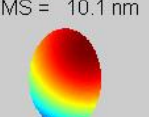

$\mathrm{RMS}=12.5 \mathrm{~nm}$

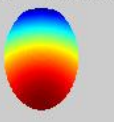

$\mathrm{RMS}=3.3 \mathrm{~nm}$

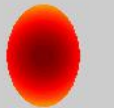

RMS $=12.4 \mathrm{~nm}$

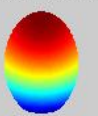

(b)

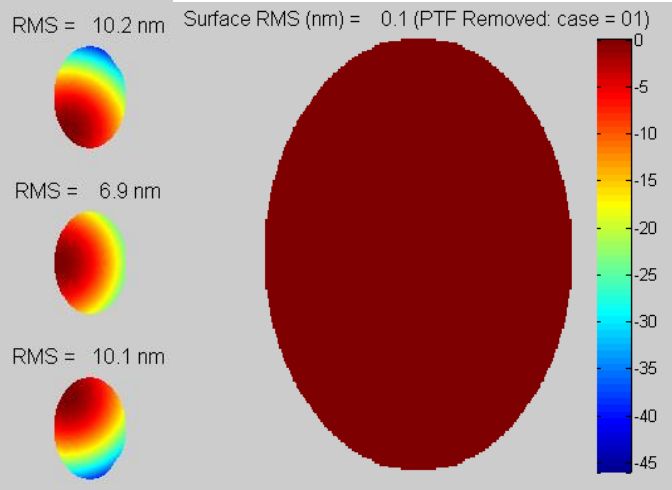

(c)

Fig. 13. (a) Thermal deformation print-through calculated over the entire optical surface before PTF corrections, (b) Thermal deformation maps of each of 9 Sub-apertures and RMS surface errors, (c) the optical thermal deformation after PTF corrections. The thermal response calculations assume M3 is at a face up position.

\section{SUMMARY AND CONCLUSION}

In order to calculate the temperature distribution of the TMT M1 assembly, M2CA, and M3CA, thermal finite element analysis was performed using extended models, which included mirror cell components for practical heat transfer boundary conditions. The thermal responses from the finite element analysis demonstrated that convection will be the most dominant mode of heat transfer, followed by radiation and conduction, respectively. For the TMT M1 assembly, the peak thermal response on the mirror under air convection and radiative heat transfer was calculated to be $2.21{ }^{\circ} \mathrm{C}$ at 7.5 hours, along with thermal gradient of $0.914^{\circ} \mathrm{C}$. From a thermo-elastic analysis with the peak thermal loading, a PV surface error of $4 \mathrm{~nm}$ was obtained over the optical surface with an RMS error of $0.3 \mathrm{~nm}$ before performing PTF corrections. After correcting pistons, tilts and focus aberrations, the PV on the optical surface reduced to $1.2 \mathrm{~nm}$ with an 
RMS error of $0.1 \mathrm{~nm}$. The thermal results of both the M3 and the M2 were very similar. The maximum optical surface displacement of M2 was found at $7.2 \mathrm{~nm}$ with an RMS surface error of $2.2 \mathrm{~nm}$. With M2 active optics, the optical surface PV error was reduced to $1.2 \mathrm{~nm}$ and RMS to $0.3 \mathrm{~nm}$ after piston, tilts, and focus corrections. The tertiary mirror demonstrated greater displacement, reaching maximums of around $49.0 \mathrm{~nm}$ normal to its front surface. With M3 active optics, the optical surface was corrected almost entirely after piston, tilts, and focus corrections.

In future studies, the thermal responses of the TMT optics will be explored for additional environmental cases. These cases will include the effects of constant heat loads, continuous operation periods extended from the 24 hours, and ambient air profiles from other site tests. Additionally, establishing an integrated thermal FE model of the TMT telescope with the optics assemblies would be highly desirable to evaluate the overall thermal performance of the TMT. This will provide an opportunity to quantify thermal effects of each sub-assembly, thermal interaction, and the global nature of the thermal behavior of the TMT.

\section{ACKNOWLEDGMENTS}

This research was carried out at the National Optical Astronomy Observatory, and was sponsored in part by the TMT. The authors gratefully acknowledge the support of the TMT partner institutions. They are the Association of Canadian Universities for Research in Astronomy (ACURA), the California Institute of Technology and the University of California. This work was supported as well by the Gordon and Betty Moore Foundation, the Canada Foundation for Innovation, the Ontario Ministry of Research and Innovation, the National Research Council of Canada, the Natural Sciences and Engineering Research Council of Canada, the British Columbia Knowledge Development Fund, the Association of Universities for Research in Astronomy (AURA) and the U.S. National Science Foundation.

The authors would like to acknowledge Laurie Phillips of the GSMT Program Office of NOAO and Ben Platt, Curtis Baffes, Eric Williams, and Larry Stepp of the TMT Project for their review and helpful comments.

\section{REFERENCES}

[1] Mast, T. and Nelson, J., “TMT Image Size and Wavefront Error Budgets Volume 1 2, 3”, TMT.OPT.TEC.07.001, (2007)

[2] Cho, M. and Corredor, A., "TMT M1 Segment Thermal Analysis", TMT.SEN.TEC.06.034.DRF01, December 12, (2006).

[3] Ponchione, R.J., Ponslet, E., Setoodeh, S., Stephens, V., Tubb, A. and Williams, E., "TMT M1 Segment Support Assembly (SSA) Preliminary Design Review (PDR)”, IMTEC, TMT.OPT.PRE.07.056.REL01, October 24, (2007)

[4] Cho, M. and Corredor, A., "Thermal Analysis of TMT M1 Segment Assembly", TMT.SEN.TEC.08.021.DRF01, December 12, April, (2008).

[5] Cho, M. and Pootrakul, S., "Thermal Analysis of TMT Secondary Mirror Assembly and Tertiary Mirror Assembly,” TMT.SEN.TEC.08.022.DRF01, April, (2008).

[6] Vogiatzis, K., "Advances in Aerothermal Modeling for TMT”, SPIE 7017-29, Marseille, France, (2008).

[7] Schöck, M., et al., "Status of the Thirty Meter Telescope site selection program", SPIE 7012-68, Marseille, France, (2008).

[8] Dalrymple, N.E., “ATST Primary Mirror Thermal Analysis: Zero-Dimensional, Time-Dependent Model”, ATST Project, April, (2002).

[9] TMT Optics Group, “TMT M2 System Design Requirement Document (DRD)”, TMT.OPT.DRD.07.004.CCR28, (2007).

[10] TMT Optics Group, “TMT M3 System Design Requirement Document (DRD)”, TMT.OPT.DRD.07.005.CCR28, (2007). 